\title{
Leverage, Uncertainty and Investment Decisions *
}

\author{
Turalay Kenc \\ University of Cambridge \\ Ciaran Driver \\ SOAS University of London
}

December 18, 2019

\begin{abstract}
We explore the role of taxes on stimulating investment decisions for levered firms under cash flows and investment costs uncertainty using the adjusted present valuebased real options approach developed by Myers \& Read (2019). We extend their work to consider combined tax credits and uncertain investment costs. We then run a numerical analysis to quantify the impact of uncertainty, corporate tax and investment tax credit in stimulating investments.
\end{abstract}

Keywords: Irreversible Investment, Tax Incentives and Uncertainty, Leverage

${ }^{*}$ The authors wish to thank Ping Chen for his research assistance helpful comments on an earlier draft of the paper without implicating them for any remaining errors. 


\section{Introduction}

Myers \& Read (2019) observe that "the effects of financial leverage and taxes on the valuation of real options have never been addressed". To this end, we explore the impact of leverage and taxes on irreversible investment decisions under cash flows and investment costs uncertainty using their adjusted present value-based real options approach. Given the capital structure, which is summarized by a target debt ratio, they value contingent claims in a simple way. We extend their work to consider combined tax credits and uncertain investment costs. The latter, suggested by them as well, enables us to capture the case where investment cost correlates negatively with cash flows. This is a striking fact for many developing countries whose downturns correspond to lower growth rates of cash flows and higher prices of (imported) investment goods. Cash flow uncertainty interacts with the tax rules since firms may not be able fully utilize tax losses. The latter, distinct effect is also considered below.

An investment opportunity is a call option on its future cash flows. Firms usually have a target debt in financing such opportunities. In addition to this explicit one, there exists another leverage related to the real call option. The difference between the explicit debt and the options leverage is the debt capacity of this real call option. This capacity is usually a negative one for call options. Myers \& Read (2019) show that "negative debt capacity means that the options leverage displaces explicit borrowing". To the extent that it displaces explicit debt, it will reduce the value of the tax shields. In this paper, we will derive their expressions and compute their values.

\section{Investment Policy}

We consider a risk-neutral firm which operates in complete markets in which time evolves continuously. The firm faces a standard irreversible investment problem to choose the 
timing of investment optimally under uncertainty and taxes. This investment project generates future uncertain cash flows over an infinite horizon once undertaken. If the project is unsuccessful, its fixed cost that is paid upfront is unrecoverable. To resolve uncertainty, the investor may delay the investment decision, which constitutes an option to wait for the firm. As for taxes, we consider only corporate taxes together with the tax-deductibility of interest expenses. In this model, investors anticipate that taxes affect not only cash flows as in the conventional approach but also discount rates in this model.

We assume an economy with complete financial markets whose agents make decisions over an infinite time horizon. Agents take the probability space $(\Omega, \mathcal{F}, \mathbb{P})$ where $\Omega$ is the set of possible realizations of the economy, $\mathcal{F}$ the $\sigma$-field of distinguishable events and $\mathbb{P}$ the actual probability on $\mathcal{F}$ as given. Finally, $\mathbb{F}=\left\{\mathcal{F}_{t}, t \in[0, \infty]\right\}$ denotes the augmented filtration or information generated by the process of asset prices. As for dealing with future cash flows agents utilize stochastic discount factors which satisfy their risk-neutrality behavior. We start with a stochastic discount factor $\wedge$ to discount future cash flows:

$$
d \Lambda(t)=-\Lambda(t)\left[r d t+v d Z_{\Lambda}(t)\right],
$$

where $r$ is the risk-free rate, $v$ the market price of risk and $Z_{\Lambda}(t)$ is a standard Brownian motion under the physical measure P.

Following the literature for the sake of tractability, we model the investment project's after-tax cash flows $\pi$ as a geometric Brownian motion. ${ }^{1}$

$$
\mathrm{d} \pi(\mathrm{t})=\pi(\mathrm{t})\left[(1-\tau) \mu_{\pi} \mathrm{dt}+\left(1-\tau^{\prime}\right) \sigma_{\pi} \mathrm{d} Z_{\pi}(\mathrm{t})\right], \quad \pi(0)=\pi_{0}
$$

where $\mu_{\pi}>0$ is the mean growth rate of future cash flows, $\sigma_{\pi}>0$ the magnitude of the uncertainty and $Z_{\pi}$ denotes another standard Brownian motion under the physical measure

\footnotetext{
${ }^{1} \mathrm{~A}$ more realistic modelling of cash flows would require negative cash flows, i.e., normally distributed cash-flows. In this regards, arithmetic Brownian motion or its mean reversion extended version is better suited to model cash flows.
} 
P. Finally, the correlation between $Z_{\Lambda}(t)$ and $Z_{\pi}(t)$ is $\rho_{\pi, \Lambda}$. Having specified the processes for the discount factor and cash flows, we now define the market price of risk as $\nu=\frac{\mu_{\pi}-r}{\sigma_{\pi}}$. Also, note that the mean growth rate of cash flows defined as $\mu_{\pi}=r+v \rho\left(1-\tau^{\prime}\right) \sigma_{\pi}$, hence its risk-neutral counterpart is $\mu^{Q}=(1-\tau) \mu_{\pi}-v \rho_{\pi, \Lambda}\left(1-\tau^{\prime}\right) \sigma_{\pi}$. This gives rise to the following payout ratio:

$$
\delta=r+v \rho_{\pi, \Lambda}\left(1-\tau^{\prime}\right) \sigma_{\pi}-(1-\tau) \mu_{\pi}=r-\mu_{\pi}^{Q}
$$

where is assumed that $\delta>0$ for the value of the project to be bounded. We assume that in financing this project, the firm adopts a capital structure characterized by a target debt ratio of $\lambda$, implying that the $\lambda$ share of the total value of the firm is the debt value. Debt financing generates tax savings to the extent that its interest payments deducted from corporate taxation. Then, the present value of project value is given by

$$
\mathrm{V}^{\mathrm{PV}}(\pi, 0)=\mathrm{E}_{0}^{\mathrm{P}}\left\{\int_{0}^{\infty} \frac{\Lambda(\mathrm{t})}{\Lambda(0)} \pi(\mathrm{t}) \mathrm{dt}\right\}-c \lambda \mathrm{V}^{\mathrm{PV}}+\tau c \lambda \mathrm{V}^{\mathrm{PV}}
$$

whre $\tau$ is the corporate tax rate and $c$ is the coupon rate on the perpetual bond. Obtaining equations for $\Lambda(t)$ and $\pi(t)$ from Eq. (1) and Eq. (2), respectively and plugging them into Eq. (3) and taking expectation and integrating the resulting equation yields:

$$
\mathrm{V}^{\mathrm{PV}}(\pi, 0)=\frac{1}{1+(1-\tau) \mathrm{c} \lambda} \frac{\pi(0)}{\delta}
$$

where $1 / \delta$ is the price-earnings ratio.

Upon the investment project initiation, say at time $t=0$, the firm starts to generate perpetual stochastic cash flows. It is assumed that the firm faces stochastic investment costs for several reasons: (i) changes in technological and market conditions; (ii) the impact of macroeconomic risk; and (iii) the introduction of investment tax credit stimulus. The first point can be captured by a GBM type of uncertainty modelling for investment costs as 
follows:

$$
d I(t)=\mu_{I} I(t) d t+\sigma_{I} I(t) d Z_{I}
$$

where $I(t)$ is the cost of investment at time $t, \mu_{I}$ the mean growth rate of investment costs, $\sigma_{I}$ the volatility term associated with the investment cost and $Z_{I}$ the usual Brownian motion term which correlates with both that of the cash flows whose correlation coefficient denoted by $\rho_{\pi, \mathrm{I}}$ and stochastic discount factor denoted by $\rho_{\mathrm{I}, \Lambda}$. Since this is a one-off investment, only uncertainty over future cash flows has an impact on the value of the project. So, the net present value of the investment project is equal to classical NPV:

$$
\mathrm{V}^{N P V}(\pi, 0)=\frac{1}{1+(1-\tau) c \lambda} \frac{\pi(0)}{\delta}-\left(1-\tau_{\mathrm{I}}\right) \mathrm{I}=\mathrm{V}^{\mathrm{PV}}-\left(1-\tau_{\mathrm{I}}\right) \mathrm{I}
$$

where $1 / \delta$ is the price-earnings ratio and $\tau_{I}$ is the investment tax credit. This equation, as expected, shows that only the cash flow uncertainty has an impact on the present value of the project. However, both investment costs and cash flows uncertainties will affect the project's option embedded value.

Given uncertainty and irreversibility, the firm chooses the timing of its investment time optimally. So, the firm's control variable is the decision about when to invest in the project. However, the firm's state variables are $\pi$ and I. So the manager effectively chooses the cash flow and investment cost levels at which it is optimal to invest. To simplify the problem, we use the ratio of $\pi$ and I denoted by $y$. When $y$ reaches the value of $y_{1}$, the firm will invest. To facilitate the solution, we utilize an Arrow-Debreu claim $A\left(\pi(0), \pi_{\mathrm{I}}\right)$. This fictitious asset pays exactly $\$ 1$ when the new process $y(t)$ starting at $y(0)$ reaches $y_{I}$ for the first time 
from below, and 0 otherwise. The value of the project before investing is then given by: ${ }^{2}$

$$
\begin{aligned}
F(y, 0) & =A\left(y(0), y_{I}\right) I\left[\frac{1}{1+(1-\tau) c \lambda} \frac{y_{I}}{\delta}-\left(1-\tau_{I}\right)\right] \\
& =\overbrace{A\left(y(0), y_{I}\right)}^{\text {effect of discounting }} \times \underbrace{V^{N A P V}\left(y_{I}, 0\right)}_{\text {size of net benefit }}
\end{aligned}
$$

where the variable $y$ is defined exclusive of investment tax credit such that $y=\pi / \mathrm{I}$. As mentioned in Dixit et al. (1999) the investment decision is as a trade-off between the size of the net benefit and the effect of discounting.

We obtain an expression for the value of the Arrow-Debreu security by solving the following ordinary differential equation (ODE):

$$
E_{0}^{P}\left\{d\left\langle A\left(y(0), y_{I}\right), \Lambda_{t}\right\rangle\right\}=0: \quad \frac{1}{2} \wedge A_{y y}(d y)^{2}+\Lambda A_{y} d y+A_{y} d y d \Lambda+A d \Lambda=0
$$

and its boundary conditions are

$$
A\left(y(0), y_{I}\right)= \begin{cases}A & y(0)<y_{I} \\ 1 & y(0) \geqslant y_{I}\end{cases}
$$

Using Ito's lemma (omitting time indexes and the arguments of $A$ ) and after simplifying, we explicitly write Eq. (8) as:

$$
\frac{1}{2} \sigma_{y}^{2} y^{2} A_{y y}+\left[\mu_{I}^{Q}-\mu_{\pi}^{Q}\right] y A_{y}-\mu_{I}^{Q} A-r A=0
$$

where we have two new terms $\sigma_{y}$ and $\mu_{\mathrm{I}}^{\mathrm{Q}}$ which are given by

$$
\begin{aligned}
\sigma_{y} & =\sqrt{\left(1-\tau^{\prime}\right)^{2} \sigma_{\pi}^{2}-2 \rho_{\pi, \mathrm{I}}\left(1-\tau^{\prime}\right) \sigma_{\pi} \sigma_{\mathrm{I}}+\sigma_{\mathrm{I}}^{2}} \\
\mu_{\mathrm{I}}^{\mathrm{Q}} & =\mu_{\mathrm{I}}-\rho_{\mathrm{I}, \Lambda} \nu \sigma_{\mathrm{I}}
\end{aligned}
$$

\footnotetext{
${ }^{2}$ This is the extended version of Eq .(4) in McDonald \& Siegel (1986).
} 
We postulate a solution for the ODE in Eq. (8):

$$
A=c_{1} y^{\gamma_{1}}+c_{2} y^{\gamma_{2}}
$$

where $c_{1}$ and $c_{2}$ are constants which will be pinned down by the above boundary conditions and $\gamma_{1}$ and $\gamma_{2}$ will be obtained from the quadratic equation associated with the above ODE equation. Plugging this back into Eq. (8) yields the well-known quadratic equation which can be solved for $\gamma_{1}$ and $\gamma_{2}$ :

$$
\gamma_{1,2}=-\left(\frac{\mu_{\pi}^{\mathrm{Q}}-\mu_{I}^{\mathrm{Q}}}{\sigma_{y}^{2}}-\frac{1}{2}\right) \pm \sqrt{\left[\frac{\mu_{\pi}^{\mathrm{Q}}-\mu_{I}^{\mathrm{Q}}}{\sigma_{y}^{2}}-\frac{1}{2}\right]^{2}+\frac{2\left(\mathrm{r}-\mu_{I}^{\mathrm{Q}}\right)}{\sigma_{y}^{2}}},
$$

By applying the boundary condition, we set $c_{2}$ and take only the positive $\gamma$, i.e., $\gamma_{1}$. The well-known value-matching and smooth-pasting conditions give us the desired expression for the Arrow-Debreu claim A:

$$
A\left(y(0), y_{I}\right)=\left(\frac{y(0)}{y_{I}}\right)^{\gamma_{1}}
$$

Finally, by applying the super contact (the second-order smooth-pasting) condition [see Dumas (1991)] to Eq. (7) we obtain the optimal $y_{\mathrm{I}}$ :

$$
y_{I}=\delta[1+(1-\tau) c \lambda]\left(1-\tau_{I}\right)\left(\frac{\gamma_{1}}{\gamma_{1}-1}\right)
$$

It is obvious that the combined uncertainty of cash flows and investment costs is greater than the standalone cash flow volatility and hence implies higher critical values. However, a greater covariance $\rho_{\pi, \mathrm{I}}$ between changes in $\pi$ and I implies less uncertainty over their ratio, and hence a reduced incentive to wait. ${ }^{3}$

\footnotetext{
${ }^{3}$ Note that the random walk modeling of the prices of physical assets is not satisfactory as they have to converge to their equilibrium levels, but not grow exponentially as suggested by a geometric Brownian motion.
} 


\section{Numerical Analysis}

We take empirically plausible values for the key parameters: $r=5 \%, \mu_{\pi}=2 \%, \mu_{\mathrm{I}}=$ $4 \%, \sigma_{\pi}=20 \%, \sigma_{\mathrm{I}}=25 \%, \rho_{\Lambda, \pi}=0.25, \rho_{\Lambda, \mathrm{I}}=0.25, \rho_{\pi, \mathrm{I}}=0.35, \tau=0.35, \tau^{\prime}=0.25, \tau_{\mathrm{I}}=$ $0.25, v=0.75, c=8 \%$ and $\lambda=0.5$. Plugging these values will give us the values of the optimal level of cash flow, the real option and the project's NPV as well as the debt capacity of the real option, explicit leverage and implicit leverage. To calculate the option's debt capacity, we use the following value matching condition:

$$
\delta^{\mathrm{OP}} \mathrm{V}^{\mathrm{PV}}-\mathrm{D}^{\mathrm{OP}}=\mathrm{V}^{\mathrm{NPV}}
$$

where $\delta^{\mathrm{OP}}$ is the usual option hedge ratio (delta), i.e., $\delta^{\mathrm{OP}}=\frac{\partial \mathrm{V}}{\partial y}$ and $\mathrm{D}^{\mathrm{OP}}$ the implicit leverage (the leverage required to replicate the real option). We derive $\delta^{\mathrm{OP}}$ from our the $\gamma$ term, which is simply the elasticity of the option value $F$ to the project value $V^{P V}$. Hence, we write

$$
\gamma=\delta^{\mathrm{OP}} \frac{\mathrm{V}^{\mathrm{PV}}}{\mathrm{F}} \Rightarrow \delta^{\mathrm{OP}}=\frac{\mathrm{F}}{\mathrm{V}^{\mathrm{PV}}} \gamma,
$$

and solve for $\mathrm{D}^{\mathrm{OP}}$. After grossing up, it is given by:

$$
\mathrm{D}^{\mathrm{OP}}=\left[\left(\frac{\mathrm{F}}{\mathrm{V}^{\mathrm{PV}}} \gamma-1\right) \mathrm{V}^{\mathrm{PV}}+\left(1-\tau_{\mathrm{I}}\right) \mathrm{I}\right] \mathrm{e}^{\mathrm{r} \tau}
$$

Finally, the debt capacity of the real option is the difference between the explicit leverage $\lambda \delta^{\mathrm{OP}} \mathrm{V}^{\mathrm{PV}}$ and the implicit leverage $\mathrm{D}^{\mathrm{OP}}$.

We reported the results of our numerical analysis in Table 1. The results highlight that the critical value of $\pi /$ I takes its lowest level when firms enjoy full offsetting tax losses, levered capital structure and investment tax credits. Accordingly, we obtain the worst case for investing when the opposites are in place. The $\pi / \mathrm{I}$ value nearly doubles from its best value 0.78 to its worst 1.46 . The results underscore the importance of the covariance of 
Table 1: Tax Policy and Investment

\begin{tabular}{|c|c|c|c|c|c|c|}
\hline & & & \multicolumn{2}{|c|}{ Correlation $\rho_{\pi, \mathrm{I}}<0$} & \multicolumn{2}{|c|}{ Correlation $\rho_{\pi, \mathrm{I}}>0$} \\
\hline & \multicolumn{2}{|c|}{$\lambda=0 \quad \lambda=0.5$} & $\lambda=0$ & $\lambda=0.5$ & $\lambda=0$ & $\lambda=0.5$ \\
\hline & \multicolumn{2}{|c|}{$\sigma_{\mathrm{I}}=0$} & \multicolumn{2}{|c|}{$\sigma_{\mathrm{I}}=25 \%$} & \multicolumn{2}{|c|}{$\sigma_{\mathrm{I}}=25 \%$} \\
\hline & \multicolumn{6}{|c|}{ Full Use of Tax Losses } \\
\hline Critical Value & 0.89 & 0.87 & 0.96 & 0.94 & 0.80 & 0.78 \\
\hline Option Value & 0.40 & 0.42 & 0.40 & 0.42 & 0.41 & 0.44 \\
\hline NPV & 0.85 & 0.81 & 0.98 & 0.94 & 0.69 & 0.65 \\
\hline \multirow[t]{2}{*}{ Debt Capacity } & -0.66 & -0.47 & -0.55 & -0.35 & -0.95 & -0.77 \\
\hline & \multicolumn{6}{|c|}{ Partial Use of Tax Losses } \\
\hline Critical Value & 0.99 & 0.97 & 1.10 & 1.07 & 0.86 & 0.84 \\
\hline Option Value & 0.41 & 0.42 & 0.42 & 0.44 & 0.40 & 0.42 \\
\hline NPV & 1.04 & 0.99 & 1.22 & 1.17 & 0.80 & 0.76 \\
\hline \multirow[t]{2}{*}{ Debt Capacity } & -0.52 & -0.32 & -0.43 & -0.22 & -0.73 & -0.54 \\
\hline & \multicolumn{6}{|c|}{$\tau_{\mathrm{I}}=0$} \\
\hline Critical Value & 1.32 & 1.29 & 1.46 & 1.43 & 1.15 & 1.12 \\
\hline Option Value & 0.31 & 0.32 & 0.33 & 0.34 & 0.28 & 0.28 \\
\hline NPV & 0.79 & 0.74 & 0.97 & 0.92 & 0.55 & 0.51 \\
\hline Debt Capacity & -0.39 & -0.24 & -0.34 & -0.18 & -0.50 & -0.36 \\
\hline
\end{tabular}

investment costs with cash flows. A positive correlation can overturn the negative impact of investment cost uncertainty. Critical values leading to investment in the last two columns are lower than those reported on the previous four columns. Calibrating the model to levered firms generates moderate gains in lower critical values. A levered firm has, on average, about 3 per cent lower level than all-equity firms. Implicit costs of real options are higher under positively correlated uncertain investment costs and reduce the value of tax shields. Our results also highlight that under negatively correlated investment costs, the net benefit has a greater role in determining values. Otherwise, the decline in the discount factor is more important.

Top panel and bottom panel of Table 1 show the sensitivity of the results to a range of volatility values as well as to tax and invest tax credit rates. High cash flow volatility combined with low investment cost volatility produces the worst outcome in terms of critical value irrespective of their correlation. Positive covariance improves the result. 
Under negative correlation, we observe some nonlinearities. As for taxes, the combination of corporate tax and investment tax credit produces the most attractive critical values for levered firms.

\section{Conclusion}

We extended the model of Myers \& Read (2019) to perpetual real options frameworks. We analyzed the impact of leverage, investment tax credit and tax shields on investment decisions. We find that investment decisions should take into account additional uncertainty and its correlation with cash flows. Policymaker should consider the use of investment tax credit in stimulating investments.

\section{References}

Dixit, A., Pindyck, R. S. \& Sødal, S. (1999), 'A Markup Interpretation of Optimal Investment Rules', 109(455), 179-189.

Dumas, B. (1991), 'Super contact and related optimality conditions', 15(4), 675-685.

McDonald, R. \& Siegel, D. (1986), 'The value of waiting to invest', 101(4), 707-727.

Myers, S. C. \& Read, J. A. (2019), 'Real Options, Taxes and Financial Leverage', 8. 
Figure 1: Effects of Correlated Cash Flows and Investment Costs on Investment
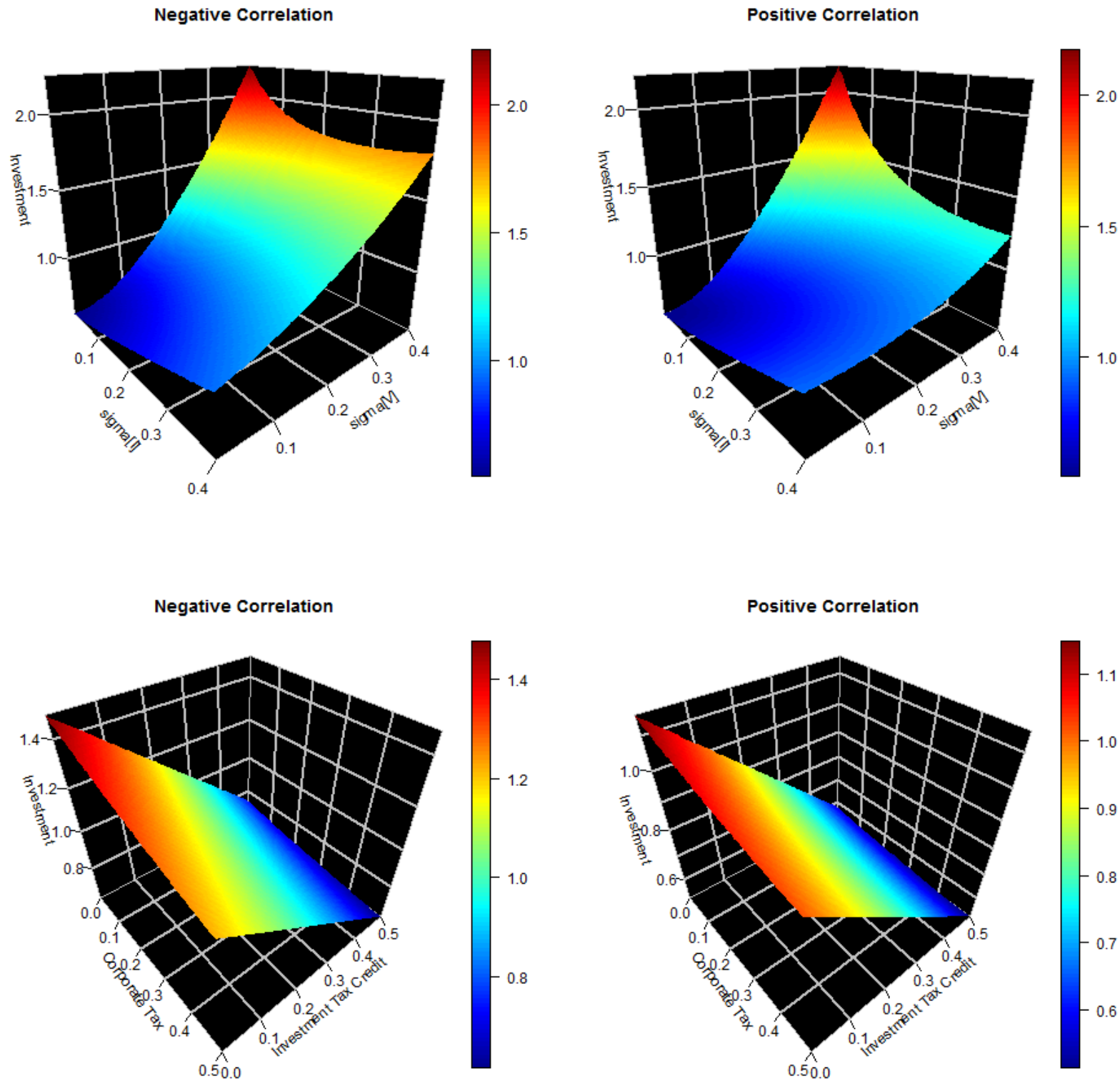